\section{Morte neonatal precoce segundo complexidade hospitalar e rede SUS e não-SUS na Região Metropolitana de São Paulo, Brasil}

\author{
Early neonatal mortality according to level of \\ hospital complexity in Greater Metropolitan \\ São Paulo, Brazil
}

Zilda Pereira da Silva 1,2 Márcia Furquim de Almeida 2 Luís Patrício Ortiz 1 Gizelton Pereira Alencar 2 Airlane Pereira Alencar 3 Daniela Schoeps 2 Elaine Garcia Minuci 1 Hillegonda Maria Dutilh Novaes 4

\author{
${ }^{1}$ Fundação Sistema Estadual \\ de Análise de Dados. \\ São Paulo, Brasil. \\ 2 Faculdade de Saúde \\ Pública, Universidade de \\ São Paulo, São Paulo, Brasil. \\ 3 Instituto de Matemática e \\ Estatística, Universidade de \\ São Paulo, São Paulo, Brasil. \\ ${ }^{4}$ Faculdade de Medicina \\ Universidade de São Paulo, \\ São Paulo, Brasil. \\ Correspondência \\ Z. P. Silva \\ Fundação Sistema Estadual \\ de Análise de Dados. \\ Av. Cásper Líbero 464, \\ São Paulo, SP \\ 01033-000, Brasil. \\ zildapereira@usp.br
}

\begin{abstract}
The aim of this study was to analyze the profile of newborns, mothers, and early neonatal mortality according to the hospital's complexity and affiliation (or lack thereof) with the Unified National Health System (SUS) in Greater Metropolitan São Paulo, Brazil. The study was based on data for live births, deaths, and hospital registries. Factor and cluster analysis were used to obtain the typology of hospital complexity and user profile. The SUS treats more high-risk newborns and mothers with low schooling, insufficient prenatal care, and teenage mothers. The probability of early neonatal death was 5.6\%o live births (65\% higher in the SUS), with no significant differences by level of hospital complexity, except those with extremely high (SUS) and medium (nonSUS) complexity. The difference in early neonatal mortality between the two systems was smaller in the group of newborns with birth weight $<1,500 \mathrm{~g}$ (22\%), but the rate was $131 \%$ higher in the SUS for newborns $\geq 2,500 \mathrm{~g}$. There was a concentration of high-risk births in the SUS, but the difference in early neonatal mortality between SUS and non-SUS hospitals was smaller in this group of newborns. New studies are needed to elucidate the high mortality rate among newborns with birth weight $\geq 2,500 \mathrm{~g}$ in the SUS.
\end{abstract}

Early Neonatal Mortality; Single Health System; Health Services
Nas últimas décadas, a mortalidade infantil decresceu significativamente em muitas regiões do mundo, porém de forma heterogênea entre os países 1. O Brasil também apresenta tendência de queda, embora se mantenha em patamares elevados, com alto grau de heterogeneidade entre as regiões. Dados estimados pela Rede Interagencial de Informações para a Saúde (RIPSA. Indicadores e dados básicos - Brasil - 2007. http://tabnet.datasus.gov.br/cgi/idb2007/matriz. htm\#mort, acessado em 28/Jul/2009) indicam que, em 2005, a taxa de mortalidade infantil correspondia a 21,2 óbitos por mil nascidos vivos, com a neonatal respondendo por cerca de $2 / 3$ das mortes infantis (14,1 por mil). A taxa de mortalidade neonatal da Região Metropolitana de São Paulo (6,0 por mil nascidos vivos) pode ser considerada elevada, quando comparada àquelas de países desenvolvidos, como Inglaterra e Canadá, que não ultrapassam a 4 por mil nascidos vivos 2. Na Região Metropolitana de São Paulo, o componente precoce responde por $70 \%$ da mortalidade neonatal e $45 \%$ da infantil. $\mathrm{O}$ aumento da proporção dos óbitos neonatais precoces e predominantemente nas primeiras 24 horas de vida estabelece uma relação mais estreita com a assistência ofertada à gestante e ao neonato no ambiente hospitalar. Impõe-se, dessa maneira, a necessidade de estudar a qualidade da assistência ao parto e ao recém-nascido, investigando o papel da assistência no processo de determinação da mortalidade neonatal 3. 
A mortalidade na primeira semana de vida é o componente da mortalidade infantil de mais difícil redução, pois resulta de complexa cadeia causal em que condições adversas da mãe e do recém-nascido contribuem para o aumento do risco de morte e para a necessidade de maior complexidade da atenção hospitalar. A literatura aponta peso ao nascer e prematuridade como os principais preditores de mortalidade neonatal 4,5,6,7,8,9 e, entre as características maternas, idades extremas, baixo nível de escolaridade, ausência de companheiro, alta paridade e presença de doenças na gravidez, como hipertensão e sangramento, constituem-se nos principais fatores de risco 3,8,9. Em relação aos serviços de saúde, a importância do pré-natal está amplamente comprovada, bem como o acesso e qualidade da atenção ao parto e ao recém-nascido 3,4,5,10,11.

Quanto à atenção hospitalar para mães e recém-nascidos, pesquisas indicam a importância da disponibilidade de leitos de UTI 12,13,14. Outros estudos valorizam características dos hospitais, como vínculo com o Sistema Único de Saúde (SUS) 3,5,11,15, complexidade da estrutura 13, atividades de ensino $3,13,15$ ou cuidado na atenção ao parto ${ }^{12}$. A complexidade da rede hospitalar tem sido estudada internacionalmente por meio de indicadores que se baseiam na presença de leitos de cuidados intensivos neonatais, volume de partos, referência para partos de alto risco e regionalização da assistência 16,17.

A análise da utilização de serviços de saúde mostra-se complexa, pois essa resulta de múltiplas determinações, como características da organização da oferta, perfil sócio-demográfico e epidemiológico dos usuários, além de aspectos relacionados à forma de financiamento e aos prestadores de serviços 18 . O presente estudo objetiva analisar características dos hospitais que atendem ao parto na Região Metropolitana de São Paulo, descrever o perfil de mães e recémnascidos e obter a probabilidade de morte neonatal precoce por complexidade dos hospitais e vínculo com o SUS.

\section{Métodos}

Estudo baseado na vinculação de dados de nascimentos, óbitos e cadastro de estabelecimentos de saúde, que engloba os hospitais que realizaram partos na Região Metropolitana de São Paulo. A população de estudo compreende os nascidos vivos ocorridos em hospitais no período de primeiro de janeiro de 2006 a 30 de junho de 2006 e os óbitos neonatais precoces pertencentes a essa coorte que ocorreram entre primeiro de janeiro de 2006 e 6 de julho de 2006.
Para os eventos vitais, foi utilizada a base de dados unificada gerada a partir de dados coletados pela Fundação Sistema Estadual de Análise de Dados (Fundação Seade) nos cartórios do registro civil e dos dados do Sistema de Informações sobre Mortalidade (SIM) e do Sistema de Informações sobre Nascidos Vivos (SINASC) das Secretarias Municipais de Saúde recebidos pela Secretaria de Estado da Saúde 4,19. Foram realizadas buscas nos documentos para complementar informações, quando necessário. Para vinculação dos dados, foi empregada técnica determinística específica, utilizada na Fundação Seade 4,20. Essa rotina contempla quatro etapas: (1) seleção, padronização e geração de variáveis derivadas; (2) definição de critérios de vinculação; (3) formação de pares por igualdade ou semelhança e (4) busca nominal nas bases de dados e documentos originais. A porcentagem de relacionamento foi elevada (99,8\%). O preenchimento dos campos nome da criança (obtido nas informações provenientes dos cartórios de registro civil) e nome da mãe e o trabalho de padronização dos nomes foram responsáveis diretos por esse resultado, equiparando-o àqueles verificados em países como os Estados Unidos 21. Dessa base inicial, foram excluídos 490 partos fora de estabelecimentos de saúde ou em local ignorado.

Para identificação do estabelecimento de nascimento, utilizaram-se dados do Cadastro Nacional de Estabelecimentos de Saúde (CNES), do Ministério da Saúde, referentes à situação em dezembro de 2005. A linkage dos registros de nascidos vivos com o CNES foi realizada a partir do código do estabelecimento da Declaração de Nascido Vivo (DNV) e, na sua ausência, do nome e do endereço do estabelecimento de saúde (campos 7 e 8 da DNV). Após a vinculação automática e busca nos documentos para os casos de código não preenchido e correção de códigos errados, foi possível vincular 99,3\% dos nascimentos institucionais aos respectivos serviços de saúde cadastrados. Após a vinculação, identificou-se que 1.450 nascimentos ocorreram em outro tipo de estabelecimento (pronto-socorro, unidade mista de saúde etc.) e foram eliminados do estudo.

Foram ainda excluídos 13 recém-nascidos que não pertenciam à Região Metropolitana de São Paulo, 45 partos domiciliares ou que ocorreram no trajeto, mas que estavam na base original como hospitalares, e 1.188 sem identificação do estabelecimento de nascimento. Após a vinculação com o CNES, foram eliminados 262 nascidos vivos de 16 hospitais que realizaram menos de 100 partos/ano 14 (cerca da metade realizou até 12 partos/ano). Foram excluídos também 2.487 registros com peso ao nascer ignorado. Verificouse que os nascidos com peso ignorado concen- 
travam-se em alguns estabelecimentos. Assim, para evitar viés na análise do perfil da clientela, decidiu-se pela exclusão dos recém-nascidos de estabelecimentos que apresentassem $25 \%$ ou mais de peso ao nascer ignorado (1.910 recémnascidos). Foram excluídos 44 recém-nascidos com peso inferior a $500 \mathrm{~g}$, em razão da baixíssima viabilidade 22 , e 22 registros referentes a óbito por anencefalia. Após o conjunto de exclusões (7.908), dos 160.651 nascidos vivos, permaneceram, no estudo, 152.743 e 861 óbitos neonatais precoces ocorridos em 143 hospitais.

Os hospitais foram classificados segundo nível de complexidade com base nas variáveis do CNES: existência de UTI neonatal com, pelo menos, seis leitos, UTI adulto, atividade de ensino (inclui hospital universitário, unidade escola superior isolada e unidade auxiliar de ensino) e referência para parto de alto risco. Foi empregada também a variável volume anual de partos, obtida da base de dados vinculada, e categorizada em: baixo (menos de 1.000 partos/ano), médio (de 1.000 a 2.999 partos/ano) e alto (3.000 e mais partos/ano) 14. Para obtenção dos grupos, com base em todas essas variáveis, utilizou-se a técnica de análise de agrupamentos 23 . Após a geração dos grupos, os hospitais foram separados em dois blocos, segundo o vínculo com o SUS, para atenção ao parto: hospitais públicos e privados que prestam atendimento ao SUS e hospitais sem esse vínculo.

Para caracterizar o perfil da clientela, selecionaram-se as variáveis: proporção de recém-nascidos com menos de $1.500 \mathrm{~g}$ (muito baixo peso), proporção de recém-nascidos com peso entre $1.500 \mathrm{~g}$ e $2.499 \mathrm{~g}$, proporção de recém-nascidos com menos de 32 semanas de gestação, proporção de recém-nascidos com 32 a 36 semanas de gestação, proporção de mães com menos de 18 anos, proporção de mães com 35 anos e mais, proporção de mães com baixa escolaridade $(<8$ anos de estudo) e proporção de mães com prénatal insuficiente $(<4$ consultas). Esse ponto de corte foi adotado tendo em vista a inclusão de nascimentos de pré-termo como indicador de risco, sendo que nem todas as mães desses recém-nascidos teriam a possibilidade de realizar seis consultas ou mais de acordo com o preconizado pelo Ministério da Saúde como pré-natal adequado 24 . Foi realizada análise fatorial pelo método de componentes principais, com rotação dos eixos pelo método varimax 23 .

Para descrever as variáveis referentes à caracterização da clientela segundo os agrupamentos de hospitais, utilizaram-se as médias de cada componente obtido na análise fatorial.

$\mathrm{Na}$ análise da mortalidade, foram calculados o risco relativo e os respectivos intervalos de $95 \%$ de confiança. Adotou-se o nível de significância de 0,05 para todos os testes estatísticos. Os dados foram processados pelo programa SPSS 13.0 (SPSS Inc. ,Chicago Estados Unidos).

Preservaram-se o sigilo e a confidencialidade das informações coletadas, e foi obtida a aprovação do Comitê de Ética em Pesquisa da Faculdade de Saúde Pública da Universidade de São Paulo.

\section{Resultados}

Foram analisados 143 hospitais agrupados segundo nível de complexidade e vínculo com o SUS: complexidade I (baixa), II (média) e III (alta) nas redes SUS e não-SUS e IV (muito alta complexidade) apenas no SUS. O número de hospitais é semelhante na rede SUS (71) e não-SUS (72), porém com distribuição desigual quanto a sua complexidade. A existência de UTI neonatal é duas vezes mais freqüente na rede SUS $(60,6 \%)$ do que na não-SUS $(27,8 \%)$, concentrando-se nos hospitais de maior complexidade. Já a presença de UTI adulto apresenta proporções semelhantes nas duas redes $(76,1 \%$ e $77,8 \%)$. Verificou-se que $63 \%$ dos hospitais na rede SUS estão cadastrados como referência para parto de alto risco, enquanto, na rede não-SUS, a proporção é de $43,1 \%$. Do total de hospitais, 29 são considerados hospitais de ensino, sendo que mais de $70 \%$ integram a rede SUS como hospitais de maior complexidade (Tabela 1).

Em relação ao volume de partos, estimado pelo número de nascidos vivos, observa-se que, entre os hospitais do SUS, predominam aqueles que realizam de 1.000 a 2.999 partos/ano $(39,4 \%)$ e mais de 3.000 partos/ano (40,8\%). Já na rede não-SUS, predominam hospitais com baixo $(45,8 \%)$ e médio $(44,4 \%)$ volume. Os hospitais de baixa e média complexidade exibem menor volume de partos (menos de 1.000 partos/ano).

Os 143 hospitais estudados distribuem-se em 29 dos 39 municípios da Região Metropolitana de São Paulo, sendo 80 na capital paulista. A rede SUS conta com mais unidades de maior complexidade (49) do que a não-SUS (26). Em termos de esfera administrativa, a maioria dos hospitais da Prefeitura de São Paulo e da rede estadual, incluindo os hospitais de tipo Organizações Sociais da Saúde (OSS), concentra-se nos grupos de maior complexidade, ao passo que metade dos hospitais de administração municipal das demais localidades da Região Metropolitana de São Paulo foi classificada como de baixa complexidade.

A rede SUS respondeu por $62,6 \%$ dos nascidos vivos na Região Metropolitana de São Paulo, 
Tabela 1

Hospitais segundo vínculo com o Sistema Único de Saúde (SUS) e nível de complexidade por principais características. Região Metropolitana de São Paulo, Brasil, janeiro a junho de 2006.

\begin{tabular}{|c|c|c|c|c|c|c|c|c|}
\hline \multirow{4}{*}{$\begin{array}{l}\text { Vínculo com } \\
\text { SUS e } \\
\text { complexidade }\end{array}$} & \multirow{4}{*}{$\begin{array}{c}\text { Número de } \\
\text { hospitais) } \\
\text { (\%) }\end{array}$} & \multirow{4}{*}{$\begin{array}{c}\text { Com atividade } \\
\text { de ensino } \\
(\%)\end{array}$} & \multirow{4}{*}{$\begin{array}{l}\text { Com UTI } \\
\text { neonatal } \\
\text { [6 e mais } \\
\text { leitos] (\%) }\end{array}$} & \multirow{4}{*}{$\begin{array}{l}\text { Com UTI } \\
\text { adulto } \\
(\%)\end{array}$} & \multirow{4}{*}{$\begin{array}{l}\text { Com Serviço } \\
\text { de parto } \\
\text { de alto risco } \\
(\%)\end{array}$} & \multicolumn{3}{|c|}{ Volume de partos/ano (\%) } \\
\hline & & & & & & Menos & $1.000 \mathrm{a}$ & $3.000 \mathrm{e}$ \\
\hline & & & & & & de 1.000 & 2.999 & mais \\
\hline & & & & & & & & \\
\hline SUS & 71,0 & 29,6 & 60,6 & 76,1 & 63,4 & 19,7 & 39,4 & 40,8 \\
\hline IV: muito alta & 2,0 & 100,0 & 100,0 & 100,0 & 100,0 & 50,0 & 50,0 & - \\
\hline III: alta & 47,0 & 40,4 & 87,2 & 87,2 & 80,9 & 2,1 & 38,3 & 59,6 \\
\hline II: média & 11,0 & - & - & 100,0 & 45,5 & 54,5 & 45,5 & - \\
\hline I: baixa & 11,0 & - & - & - & - & 54,5 & 36,4 & 9,1 \\
\hline Não-SUS & 72,0 & 11,1 & 27,8 & 77,8 & 43,1 & 45,8 & 44,4 & 9,7 \\
\hline III: alta & 26,0 & 30,8 & 73,1 & 92,3 & 76,9 & 19,2 & 53,8 & 26,9 \\
\hline II: média & 31,0 & - & 3,2 & 100,0 & 29,0 & 61,3 & 38,7 & - \\
\hline I: baixa & 15,0 & - & - & - & 13,3 & 60,0 & 40,0 & - \\
\hline Total & 143,0 & 20,3 & 44,1 & 76,2 & 53,1 & 32,9 & 42,0 & 25,2 \\
\hline
\end{tabular}

valor que se eleva para $71,4 \%$ quando se exclui a capital. A rede particular respondeu por $37,3 \%$ dos neonatos, com maior proporção na capital $(42,4 \%)$. A maioria dos nascimentos $(75,6 \%)$ ocorreu em hospitais classificados como de maior complexidade, sendo $53 \%$ no SUS (IV e III) e $22,5 \%$ (III) na rede privada.

Para analisar o perfil da clientela dos hospitais, foi realizada análise fatorial, que gerou dois componentes. O primeiro compreende variáveis das características do recém-nascido: proporção de recém-nascidos com menos de $1.500 \mathrm{~g}$, proporção de recém-nascidos com peso entre $1.500 \mathrm{~g}$ e $2.499 \mathrm{~g}$, proporção de recém-nascidos com menos de 32 semanas de gestação, proporção de recém-nascidos com 32 a 36 semanas de gestação. Esse componente explica $42,8 \%$ da variância. O segundo, formado pelas variáveis que representam as características maternas - proporção de mães com menos de 18 anos, proporção de mães com 35 anos e mais, proporção de mães com baixa escolaridade ( $<8$ anos de estudo) e proporção de mães com menos de quatro consultas de prénatal -, explica 36,5\% da variância (Tabela 2). Esses componentes foram denominados de: risco do recém-nascido e risco social da mãe.

O componente risco do recém-nascido mostra escore simetricamente oposto e mais elevado para os hospitais do SUS $(0,19)$ em relação aos da rede não-SUS $(-0,18)$. Resultado semelhante foi observado para o fator risco social da mãe (escores de 0,87 no SUS e $-0,85$ na rede não-SUS). Na análise dos fatores, verificou-se que dois hospitais universitários se destacaram por apresenta- rem perfil muito distinto dos demais, especialmente em relação ao escore elevado para o fator risco do recém-nascido. Esse resultado ajudou na decisão de reclassificá-los no critério de complexidade, criando o grupo de complexidade IV - SUS (Figura 1).

A rede SUS concentra os recém-nascidos de baixo peso $(10,5 \%)$, entre os quais, $1,7 \%$ pesava menos que $1.500 \mathrm{~g}$, e $8,8 \%$, entre $1.500 \mathrm{~g}$ e $2.499 \mathrm{~g}$. Na rede privada, a prevalência de baixo peso ao nascer foi de $7,8 \%$, com $1,1 \%$ e $6,8 \%$, respectivamente nas duas categorias de peso. Essas diferenças são estatisticamente significantes. A proporção de pré-termo no SUS foi mais elevada (9\%) que na rede não-SUS $(7,7 \%)$, porém a diferença não é estatisticamente significante (Tabela 3).

$\mathrm{Na}$ análise intra-rede, o grupo de altíssima complexidade (SUS) destaca-se dos demais por apresentar maior escore do fator risco do recémnascido $(4,9)$. Esse grupo registra cerca de $31 \%$ de neonatos de baixo peso e $28 \%$ de pré-termo. Comparado com o grupo III, concentra 5 vezes mais neonatos de muito baixo peso, 4,4 vezes mais recém-nascidos muito prematuros $(<32 \mathrm{se}$ manas) e cerca de 2,5 vezes de recém-nascidos com 32 a 36 semanas de gestação e com $1.500 \mathrm{~g}$ a 2.499g ao nascer. Os hospitais classificados como de alta e média complexidade exibem perfil semelhante de risco do recém-nascido, sem diferença estatisticamente significante. Já os hospitais de menor complexidade no SUS (I) detêm mais baixas proporções de recém-nascidos de baixo peso e de pré-termo, valores sintetizados no menor escore de risco do recém-nascido 
Resultados da análise fatorial: matriz de cargas dos dois primeiros componentes rotacionados.

Proporção de recém-nascidos com peso ao nascer de menos de $1.500 \mathrm{~g}$

Proporção de recém-nascidos com idade gestacional menor que 32 semanas de gestação

Distribuição dos hospitais segundo classificação dos escores nos fatores (1) risco do recém-nascido e (2) risco social da mãe.

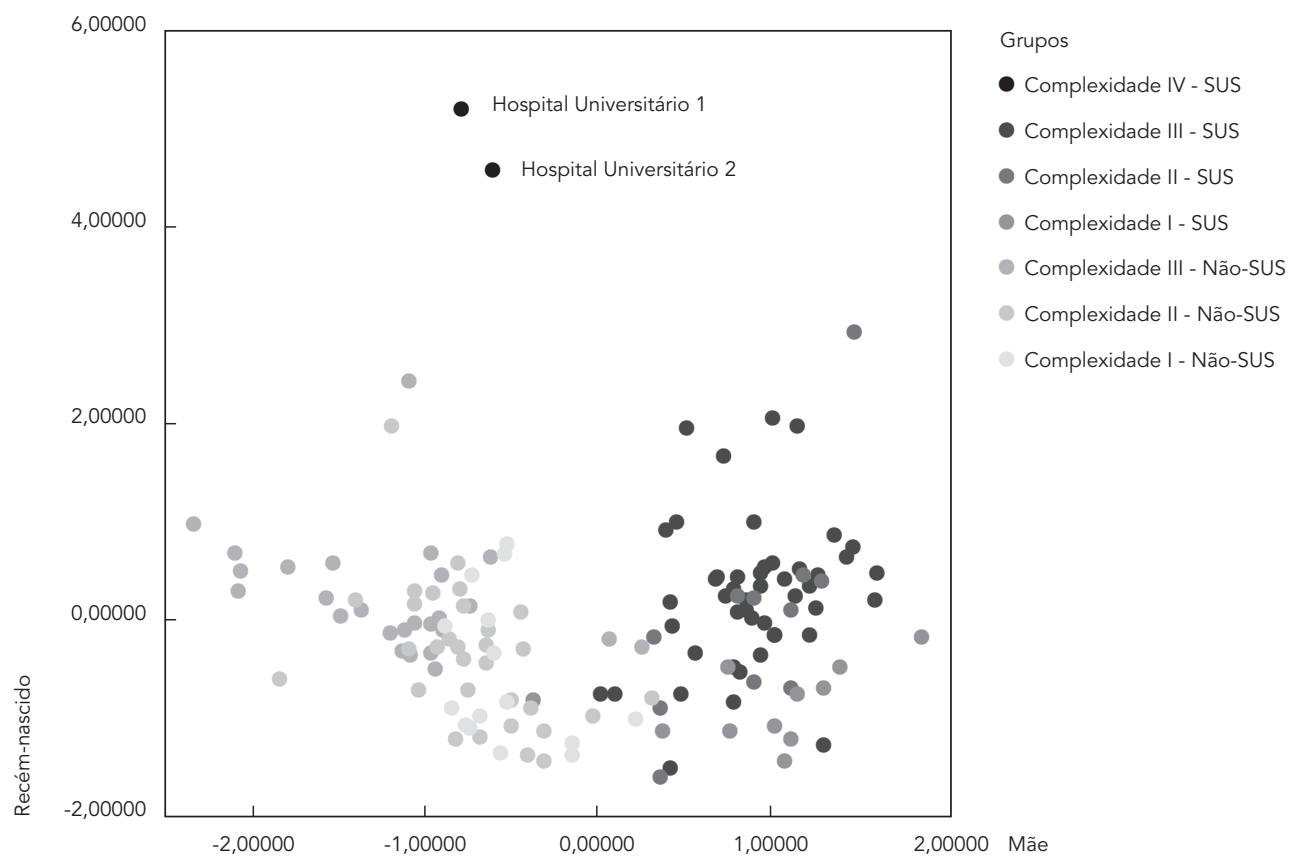

$(-0,85)$ entre os sete grupos. Essas variáveis apresentaram significância estatística na comparação com o grupo III.

Entre os hospitais não-SUS, também se observa um gradiente nos escores do fator risco do recém-nascido que decrescem do grupo de alta complexidade $(0,24)$ para o de baixa complexida- de $(-0,55)$. Os de maior complexidade atenderam mais freqüentemente recém-nascidos de muito baixo peso do que os dos grupos de média e baixa complexidade. O mesmo vale para os recém-nascidos com peso entre $1.500 \mathrm{~g}$ e $2.499 \mathrm{~g}$. As freqüências dos hospitais de média e baixa complexidade são semelhantes e se diferenciam estatistica- 
Tabela 3

Fator risco do recém-nascido, características dos nascidos vivos, fator risco social da mãe e características das mães, probabilidade de morte neonatal precoce, segundo vínculo com o Sistema Único de Saúde (SUS) e nível de complexidade dos hospitais. Região Metropolitana de São Paulo, Brasil, janeiro a junho de 2006 .

\begin{tabular}{|c|c|c|c|c|c|c|c|c|c|c|c|}
\hline \multirow{2}{*}{$\begin{array}{l}\text { Vínculo com } \\
\text { o SUS e } \\
\text { complexidade }\end{array}$} & \multirow{2}{*}{$\begin{array}{l}\text { Fator } \\
\text { risco do } \\
\text { recém- } \\
\text { nascido }\end{array}$} & \multicolumn{4}{|c|}{ Proporção de nascidos vivos (\%) } & \multirow{2}{*}{$\begin{array}{c}\text { Fator } \\
\text { risco } \\
\text { social } \\
\text { da mãe }\end{array}$} & \multicolumn{4}{|c|}{ Proporção de mães (\%) } & \multirow[b]{2}{*}{$\begin{array}{c}\text { Probabili- } \\
\text { dade de } \\
\text { morte } \\
\text { (por } \\
\text { mil } \\
\text { nascidos } \\
\text { vivos) }\end{array}$} \\
\hline & & $\begin{array}{c}\text { Menos de } \\
1.500 \mathrm{~g}\end{array}$ & $\begin{array}{c}1.500 \mathrm{~g} \mathrm{a} \\
2.499 \mathrm{~g}\end{array}$ & $\begin{array}{c}\text { Menos } \\
\text { de } 32 \\
\text { semanas }\end{array}$ & $\begin{array}{c}32 \text { a } 36 \\
\text { semanas } \\
\text { de } \\
\text { gestação }\end{array}$ & & $\begin{array}{c}\text { Menos de } \\
18 \text { anos }\end{array}$ & $\begin{array}{l}\text { Mais de } \\
34 \text { anos }\end{array}$ & $\begin{array}{c}\text { Menos de } \\
8 \text { anos } \\
\text { de estudo }\end{array}$ & $\begin{array}{c}\text { Menos } \\
\text { de } 4 \\
\text { consultas }\end{array}$ & \\
\hline \multicolumn{12}{|c|}{ Vínculo com o SUS } \\
\hline SUS & 0,19 & 1,67 & 8,80 & 1,68 & 7,34 & 0,87 & 8,97 & 10,88 & 38,7 & 9,76 & 6,60 * \\
\hline Não-SUS & $-0,18$ & 1,07 * & 6,77 * & 1,21 & 6,51 & $-0,85$ & $3,11^{\star}$ & 15,36 * & 13,52 * & 1,23 * & 4,01 \\
\hline \multicolumn{12}{|l|}{ Complexidade } \\
\hline \multicolumn{12}{|l|}{ SUS } \\
\hline IV & 4,90 & $8,58 * \star$ & $22,80 \star \star$ & $7,59 * \star$ & 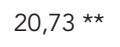 & $-0,7$ & $4,61 \star \star \star$ & $25,00 * \star$ & 31,97 & 9,37 & $34,64 * \star$ \\
\hline III & 0,26 & 1,72 & 9,05 & 1,73 & 7,97 & 0,9 & 9,29 & 10,78 & 38,74 & 9,88 & 6,22 \\
\hline$\|$ & 0,04 & 1,60 & 7,89 & 1,56 & 6,88 & 0,9 & 8,57 & 10,36 & 40,2 & 10,04 & 7,11 \\
\hline 1 & $-0,85$ & $0,30 * \star$ & $6,09 * \star$ & $0,50 * \star$ & $2,66 * \star$ & 0,96 & 8,78 & $9,28 * \star$ & 38,24 & 9,03 & 4,36 \\
\hline \multicolumn{12}{|l|}{ Não-SUS } \\
\hline III-não-SUS & 0,24 & 1,39 & 8,00 & 1,44 & 8,63 & $-1,18$ & 2,46 & 18,35 & 9,05 & 1,46 & 3,48 \\
\hline II-não-SUS & $-0,36$ & $0,91 * \star \star$ & $6,20 * \star \star$ & 1,19 & $5,86 * * \star$ & $-0,73$ & 3,24 & 13,51 *** & $14,33 * \star *$ & 1,01 & $4,92 * \star \star$ \\
\hline I-não-SUS & $-0,55$ & $0,86 * \star \star *$ & 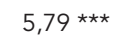 & 0,86 & 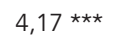 & $-0,54$ & 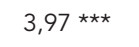 & $14,00 * * \star$ & $19,60 * * *$ & 1,27 & 4,61 \\
\hline Total & & 1,37 & 7,77 & 1,44 & 6,92 & & 6,02 & 13,14 & 26,02 & 5,46 & 5,64 \\
\hline
\end{tabular}

* $p<0,05$ na comparação com o grupo de hospitais SUS;

** $p<0,05$ na comparação entre os hospitais do SUS (referência: grupo III);

*** $p<0,05$ na comparação entre os hospitais não-SUS (referência: grupo III).

mente das do grupo III. Quanto aos pré-termos, não se observou diferença significante para a proporção de nascimentos de muito pré-termo (<32 semanas de gestação). Já em relação aos recém-nascidos com idade gestacional de 32 a 36 semanas, os hospitais do grupo de alta complexidade apresentam maior freqüência $(8,6 \%)$.

O escore do fator risco social da mãe expressa diferença oposta entre o perfil da clientela atendida pelos hospitais SUS $(0,87)$ e não-SUS $(-0,85)$. O SUS atende uma proporção quase três vezes maior de mães adolescentes do que a rede nãoSUS, em contrapartida, recebe menos mães com 35 anos e mais $(10,8 \%$ vs. $15,4 \%)$. A baixa escolaridade no SUS é quase o triplo da encontrada na rede privada. As mães com pré-natal insuficiente foram quase oito vezes mais freqüentes no SUS que na rede não conveniada $(1,2 \%)$ (Tabela 3). Todas essas diferenças foram significantes $(\mathrm{p}<0,05)$.

Na análise por complexidade, o escore do fator risco social da mãe mostra maior homogeneidade no SUS, com exceção dos dois hospitais do grupo IV, que exibem perfil muito específico, com elevadas proporções de mães com baixa escolaridade (32\%), alta proporção de pré-natal insuficiente $(9,4 \%)$, menor proporção de mães adolescentes $(4,6 \%)$ e maior presença de mães com 35 anos e mais (25\%). Esse grupo registra escore negativo para esse componente $(-0,70)$. Na comparação com as características maternas do grupo de alta complexidade do SUS, apenas as proporções relativas ao perfil etário foram estatisticamente significantes.

Ainda na rede SUS, os demais grupos (III, II e I) têm perfil semelhante em relação às características maternas, a única diferença estatisticamente significante refere-se à maior proporção de mães com 35 anos e mais que deram à luz nos hospitais de alta complexidade. $\mathrm{O}$ contingente de mães com baixa escolaridade é elevado, cerca de $39 \%$ nos três grupos. O acesso ao pré-natal também se revela mais precário; em todos os grupos, o percentual de mães que realizaram menos de quatro consultas situa-se na faixa de $9 \%$ a $10 \%$.

As mães que realizaram parto na rede privada possuem características mais heterogêneas, com o fator risco social da mãe decrescente do 
grupo I ao III (-0,54, -0,73 e -1,18), o que indica um gradiente do perfil social inverso ao nível de complexidade do hospital. A proporção de mães com menos de 18 anos é inferior nos hospitais de maior complexidade relativamente aos de menor complexidade, sendo estatisticamente significante. As mães com 35 anos e mais têm maior presença no grupo III $(18,4 \%)$ e percentuais semelhantes no II e I ( $p<0,05$ comparação como o grupo III). As mulheres atendidas nos hospitais de maior complexidade apresentam maior escolaridade do que as atendidas nos outros grupos $(\mathrm{p}<0,05)$. As proporções de pré-natal insuficiente não foram estatisticamente diferentes entre os níveis de complexidade.

A probabilidade de morte neonatal precoce hospitalar foi de 5,6 óbitos por mil nascidos vivos, sendo $65 \%$ mais elevada no SUS $(6,6 \%$ o nascidos vivos) do que na rede privada $(4,1 \%$ o nascidos vivos). Os neonatos de muito baixo peso exibem maiores probabilidades de morte tanto na rede SUS (260,4\%o nascidos vivos) como nãoSUS (214,2\%o nascidos vivos), contudo, salientase que a probabilidade observada na rede SUS é $22 \%$ maior que a da rede particular. Para os recém-nascidos de peso entre $1.500 \mathrm{~g}$ e $2.499 \mathrm{~g}$, as probabilidades não são estatisticamente diferentes. Já para o grupo de 2.500 g e mais, a probabilidade de morte na rede SUS é 2,4 vezes maior que na rede não-SUS (Tabela 4).

De forma geral, não houve diferença estaticamente significante entre os níveis de complexidade. No SUS, apenas a probabilidade de morte nos hospitais do grupo IV foi significantemente diferente $(p<0,05)$, representando um risco 5,6 vezes maior no global, 4,6 no grupo de recémnascidos de $1.500 \mathrm{~g}$ a $2.499 \mathrm{~g}$, e 5,3 vezes para os recém-nascidos com $2.500 \mathrm{~g}$ e mais (dados não apresentados). Entre os hospitais não-SUS, a probabilidade de morte neonatal precoce foi de 3,5 óbitos por mil nascidos vivos no grupo III, 4,92 no II e 4,61 no I, porém, somente para o grupo II, essa diferença é estatisticamente significante $(\mathrm{p}<0,05)$. Entre os recém-nascidos com menos de $1.500 \mathrm{~g}$, nascer nos hospitais dos grupos II e I representou risco de morte quase 2 vezes maior do que nos hospitais do grupo III. Para as demais categorias de peso, não houve diferenças significantes.

Óbitos de menores de 7 dias, probabilidade de morte neonatal precoce, segundo vínculo do hospital com o Sistema Único de Saúde (SUS), por peso ao nascer. Região Metropolitana de São Paulo, Brasil, janeiro a junho de 2006.

\begin{tabular}{|c|c|c|c|c|c|c|}
\hline $\begin{array}{l}\text { Vínculo com o SUS } \\
\text { e peso ao nascer }\end{array}$ & $\begin{array}{l}\text { Óbitos em } \\
\text { menores } \\
\text { de } 7 \text { dias }\end{array}$ & $\begin{array}{l}\text { Total de } \\
\text { nascidos } \\
\text { vivos }\end{array}$ & $\begin{array}{l}\text { Probabilidade } \\
\text { de morte } \\
\text { (por mil } \\
\text { nascidos } \\
\text { vivos) }\end{array}$ & RR & IC95\% & $\begin{array}{l}\text { Valor } \\
\text { de p }\end{array}$ \\
\hline \multicolumn{7}{|l|}{ Total } \\
\hline SUS & 632 & 95.700 & 6,6 & 1,65 & $1,41-1,91$ & 0,000 \\
\hline Não-SUS & 229 & 57.043 & 4,0 & 1,00 & & \\
\hline Total & 861 & 152.743 & 5,6 & & & \\
\hline \multicolumn{7}{|l|}{ Menos de $1.500 \mathrm{~g}$} \\
\hline SUS & 375 & 1.440 & 260,4 & 1,22 & $1,03-1,44$ & 0,0221 \\
\hline Não-SUS & 142 & 663 & 214,2 & 1,00 & & \\
\hline Total & 517 & 2.103 & 245,8 & & & \\
\hline \multicolumn{7}{|l|}{$1.500 \mathrm{~g}$ a $2.499 \mathrm{~g}$} \\
\hline SUS & 108 & 8.069 & 13,4 & 1,18 & $0,84-1,65$ & 0,3417 \\
\hline Não-SUS & 48 & 4.224 & 11,4 & 1,00 & & \\
\hline Total & 156 & 12.293 & 12,7 & & & \\
\hline \multicolumn{7}{|l|}{$2.500 \mathrm{~g}$ e mais } \\
\hline SUS & 149 & 86.191 & 1,7 & 2,31 & $1,63-3,29$ & $<0,0001$ \\
\hline Não-SUS & 39 & 52.156 & 0,7 & & & \\
\hline Total & 188 & 138.347 & 1,4 & & & \\
\hline
\end{tabular}

IC95\%: intervalo de 95\% de confiança; RR: risco relativo. 


\section{Discussão}

É reconhecida a importância de pesquisas em sistemas e serviços de saúde que podem contribuir para o aprimoramento e fortalecimento do SUS e para a qualidade na atenção nos serviços de saúde 25. No Brasil, poucos estudos investigam os diferenciais da mortalidade neonatal precoce, segundo as características do hospital de ocorrência do nascimento 4,5,15,22.

Neste trabalho, buscou-se conhecer a estruturação da rede hospitalar em termos de complexidade para a assistência obstétrica e ao recémnascido, bem como descrever o perfil das mães, dos recém-nascidos e da mortalidade neonatal precoce. O grau de complexidade da infra-estrutura, entendida como a disponibilidade em quantidade e qualidade de serviços, equipamentos, tecnologia e recursos humanos, foi analisado apenas como potencial de resolubilidade da assistência ${ }^{14}$. Não se considerou o emprego, com adequação e oportunidade, de tecnologias disponíveis em cada grupo de hospitais.

Quanto à organização da oferta de assistência ao parto e ao recém-nascido, verifica-se que a rede SUS responde por $2 / 3$ desses serviços na Região Metropolitana de São Paulo, ao passo que, nos municípios do entorno da capital, atinge $71,4 \%$. Esses resultados são semelhantes aos encontrados em outras regiões do país 11,13,26, mostrando que, mesmo na região mais rica do país, o SUS é o principal financiador da assistência obstétrica. A menor participação do SUS no Município de São Paulo deve-se ao elevado contingente da população com acesso a planos e seguros privados de saúde $(58,1 \%$ ) (Agência Nacional de Saúde Suplementar. Informações em Saúde Suplementar. http://anstabnet.ans.gov. br/deftohtm.exe?dados/TABNET_TX.def, acessado em 25/Jun/2008).

A rede SUS tem maior proporção de hospitais de alta complexidade na atenção ao parto e ao recém-nascido. Os estabelecimentos que disponibilizam leitos de UTI neonatal eram duas vezes mais freqüentes na rede SUS. Esses indicadores sugerem que a rede SUS dispõe de condições de atender adequadamente os nascimentos de risco. Além disso, a rede SUS conta com maior participação de estabelecimentos com médio e alto volume de partos/ano e concentração de atividades de ensino, sugerindo que, nesses locais, profissionais experientes prestam atendimento, fornecendo potencialmente adequada atenção ao parto e ao recém-nascido.

A análise fatorial identificou que o perfil da clientela é formado por dois componentes distintos, um representando o perfil de risco dos recém-nascidos e outro o risco social das mães. A sumarização de dados expressa pelo escore fatorial dos componentes mostra mais nitidamente a diferenciação das clientelas do que a análise das variáveis isoladas. Essa diferenciação entre rede SUS e não-SUS ficou evidente ao apresentaremse escores médios opostos em relação às características maternas e às dos recém-nascidos. Os valores negativos mostram que os hospitais da rede não-SUS atendem mães e recém-nascidos de menor risco do que o SUS.

O componente formado pelas variáveis do recém-nascido apresentou maior poder explicativo que aquele formado por variáveis descritivas do perfil materno. Contudo, é importante considerar o efeito dos elevados escores do fator risco do recém-nascido dos dois hospitais universitários de altíssima complexidade. O SINASC não apresenta informações que permitam avaliar a presença de intercorrências maternas, as quais estão diretamente associadas à mortalidade neonatal precoce 3,9. Como as variáveis utilizadas não medem diretamente o risco obstétrico e representam mais apropriadamente as características socioeconômicas maternas, que exercem um papel mais distal na cadeia causal, pode-se pensar que esse componente expressa um risco potencial. Por essa razão, esse fator foi denominado risco social da mãe.

Os escores médios do fator risco social da mãe mostram que o perfil das mães atendidas no SUS é mais homogêneo do que o dos hospitais não-SUS, que apresentam grande heterogeneidade, expressando provavelmente as características dos beneficiários dos diferentes tipos de convênios de saúde privados. Nesse segmento, o escore apresentou relação inversa ao grau de complexidade dos serviços, com mães de maior risco social sendo atendidas nos hospitais de menor complexidade.

As mulheres que deram à luz nos hospitais do SUS exibem maior risco social, elas são proporcionalmente mais jovens, menos escolarizadas e têm menos acesso ao pré-natal - perfil semelhante ao observado em estudos nos municípios de São Paulo 5 e Rio de Janeiro 11.

Na rede não-SUS, registram-se maiores proporções de mães com 35 anos e mais e freqüência reduzida de atenção pré-natal. Esse perfil se intensifica conforme aumenta o grau de complexidade dos hospitais, podendo expressar também um gradiente do poder aquisitivo de suas usuárias, ou seja, hospitais de maior complexidade atendem pessoas com maior nível de renda e de escolaridade. Estudo no Município de São Paulo mostrou existir um gradiente de aumento da participação de mães mais idosas estatisticamente significante com a redução da vulnerabilidade social 27 . 
Com relação à maior proporção de pré-natal insuficiente entre as mães atendidas no SUS, outros estudos identificaram o mesmo em populações com menor nível sócio-econômico 26 , entre adolescentes e mães sem companheiro ${ }^{9}$ e em população usuária do SUS 11. Minuci \& Almeida 27 mostraram que há tendência ascendente de mães sem assistência pré-natal com aumento da vulnerabilidade social no Município de São Paulo, bem como uma associação estatisticamente significante entre a ausência de pré-natal e a baixa escolaridade, sugerindo que este pode ser um indicador de exclusão social.

As considerações anteriores são importantes, pois algumas das complicações do recém-nascido são passíveis de previsão durante a atenção pré-natal, do que se depreende que as gestantes devam ser encaminhadas para hospitais com assistência compatível com os potenciais riscos, o que, porém, exige integração entre os serviços de pré-natal e de parto para se evitar atendimento tardio a possíveis complicações 28 .

A rede SUS apresentou risco do recém-nascido elevado. De fato, esses hospitais detêm maiores freqüências de recém-nascidos de muito baixo peso e de muito pré-termo do que a rede nãoSUS, resultados semelhantes foram encontrados em Pelotas (Rio Grande do Sul) 15, Recife (Pernambuco) 22 e Rio de Janeiro 11. O componente risco do recém-nascido apresentou tendência de aumento com o incremento da complexidade dos hospitais: os grupos de alta (III) e altíssima (IV) do SUS receberam proporcionalmente mais recém-nascidos de baixo e muito baixo peso e de pré-termos do que os grupos de média e baixa complexidade.

Nos hospitais da rede não-SUS, o fator risco do recém-nascido é bem inferior em comparação aos do SUS e também mostra um gradiente em relação à complexidade dos hospitais, o que indica que há hierarquização nos serviços do setor privado.

Os resultados mostram que a maior complexidade da rede hospitalar SUS é uma resposta ao perfil epidemiológico da demanda existente, expresso pelo componente risco social materno e risco do recém-nascido. A hierarquização observada revela o grau de organização da rede SUS na Região Metropolitana de São Paulo.

Já na rede não-SUS, os resultados sugerem que a dimensão que determina a hierarquização é a condição socioeconômica da clientela. $\mathrm{O}$ perfil das mães indicou maior predominância de usuários de melhor nível sócio-econômico nos hospitais de maior complexidade. A população de menor poder aquisitivo e que conta com planos de saúde com cobertura insuficiente de serviços permanece na rede privada enquanto não há necessidade de tratamentos mais complexos e, portanto, mais caros. Estudo de Ribeiro et al. 18 mostrou que a posse de planos de saúde não foi suficiente para garantir o acesso aos serviços de saúde para parcela dos seus beneficiários. Quando há identificação de alto risco, não é pouco usual o encaminhamento para os hospitais de alta complexidade do SUS. Já a população mais rica permanece nos serviços de maior complexidade. Essa situação denota uma interdependência entre os sistemas SUS e não-SUS, mediada pelo grau de complexidade do atendimento demandado e a respectiva capacidade de pagamento dos usuários.

A mortalidade neonatal precoce é resultante de uma complexa relação entre as condições de vida das mães e das famílias, as condições psicossociais e biológicas maternas durante a gestação e a assistência pré-natal, obstétrica e ao recém-nascido ${ }^{5}$. Este estudo não pretende avaliar diretamente o efeito das condições da atenção ao parto e ao recém-nascido na mortalidade neonatal precoce, pois, como já mencionado, os indicadores aqui estudados permitem avaliar apenas a oferta potencial dos serviços de saúde 14 e algumas características epidemiológicas dos usuários. Não foram utilizados indicadores suficientemente sensíveis ou que possibilitassem medir se esses estavam disponíveis a tempo para atender às necessidades dos usuários.

A probabilidade de morte hospitalar neonatal precoce foi $65 \%$ mais elevada nos hospitais do SUS do que na rede privada. Esses achados condizem com outras pesquisas, como aquelas realizadas em Recife 22 e Goiânia 29 , que identificaram mortalidade neonatal 2 vezes maior no SUS, e, em Pelotas, 4,1 vezes maior no hospital que atendia exclusivamente pelo SUS 15. O mesmo foi observado no Rio de Janeiro ${ }^{11}$.

A mortalidade neonatal precoce nos hospitais de elevada complexidade (grupo formado por dois grandes hospitais universitários) foi 8,6 vezes a mortalidade encontrada na rede privada e 5,2 vezes a mortalidade observada nos hospitais vinculados ao SUS, pelo fato de constituírem referência para nascimentos de altíssimo risco. Foram excluídos apenas os recém-nascidos portadores de anencefalia, os demais portadores de defeitos congênitos permaneceram no estudo, sendo que esses hospitais são referência para o atendimento ao parto de fetos com diagnóstico prévio de defeito congênito e de gestantes portadoras de doenças graves que apresentam complicações na gestação. Resultados semelhantes foram observados em Campinas ${ }^{3}$ e Goiânia 29.

Já em relação aos outros níveis de complexidade (I a III) no âmbito do SUS, inesperadamente não se observou desempenho diferenciado quan- 
to à mortalidade, apesar das diferenças de suas estruturas e do escore fatorial de risco do recémnascido. Uma explicação possível seria que, nos hospitais I e II, a mortalidade é maior do que a esperada para o perfil de risco dos usuários, e que seria compatível com o nível de complexidade do serviço, o que sugere deficiência na assistência prestada ou falhas na hierarquização, entretanto, as informações existentes são insuficientes para uma avaliação mais detalhada.

Há claro diferencial de mortalidade segundo complexidade na rede privada. Os hospitais dos grupos II e I apresentaram quase o dobro de mortalidade quando comparados aos do grupo III. Esses resultados possivelmente se devem à heterogeneidade de risco social das mães atendidas nos hospitais de menor complexidade, que não dispõem de infra-estrutura e segurança para atender recém-nascidos de maior risco, resultando em maiores probabilidades de morte. Esses resultados indicam que nem sempre a hierarquização da rede privada atende ao perfil de necessidade da demanda.

Os neonatos de muito baixo peso exibem maiores probabilidades de mortalidade neonatal precoce (245,8 por mil nascidos vivos). Esse grupo apresenta taxas mais elevadas tanto nos hospitais do SUS como nos não-SUS, porém o diferencial entre as redes é pequeno. Não se observou diferença estatisticamente significante das taxas de mortalidade entre as duas redes hospitalares para o grupo de recém-nascidos com peso entre $1.500 \mathrm{~g}$ e $2.499 \mathrm{~g}$. Esses recém-nascidos requerem, em geral, cuidados intensivos, e a análise da tipologia dos hospitais mostrou que a rede SUS é mais bem equipada em termos de disponibilidade de UTI neonatal. Esses resultados mostram que, para nascimentos considerados de alto risco, o SUS disponibiliza atenção obstétrica e ao recém-nascido semelhante àquela existente na rede não-SUS, atendendo a demanda de alto risco.

A maior diferença de mortalidade neonatal precoce entre as redes SUS e não-SUS (risco relativo - RR = 2,3) situa-se, surpreendentemente, no grupo de recém-nascidos de menor risco (peso ao nascer $\geq 2.500 \mathrm{~g}$ ), o que exige um estudo mais aprofundado. A rede SUS conta com maior participação de hospitais de alta complexidade e com elevado volume de partos/ano, portanto, era de se esperar menor mortalidade, uma vez que estudo realizado na Alemanha concluiu que a probabilidade de morte neonatal precoce para os nascidos com peso normal é menor em centros especializados com grande volume de partos do que nas maternidades pequenas 17 .

Os sistemas de informações aqui empregados não contemplam informações sobre intercor- rências na gestação (hipertensão, pré-eclampsia, problemas placentários e outros). No entanto, é possível supor sua presença, uma vez que essas freqüentemente se encontram no caminho causal dos nascimentos de baixo peso e pré-termo. Já nos nascimentos de termo e não baixo peso, é possível supor que essas intercorrências são menos freqüentes $3,5,9,12$, mas podem estar presentes e contribuir para o excesso de mortalidade neonatal precoce para os nascimentos com 2.500 g e mais, no SUS. Cabe mencionar ainda que, entre as mães usuárias do SUS, há maior proporção de assistência pré-natal insuficiente, o que também pode contribuir para ausência de detecção e tratamento oportuno dessas intercorrências.

As considerações anteriores, juntamente com o maior risco social materno averiguado nos hospitais do SUS, podem dificultar a avaliação da presença de riscos nos cuidados obstétricos e do recém-nascido. No entanto, essa explicação dá conta apenas de parte desses resultados. Lansky et al. 12, em estudo realizado em hospitais SUS de Belo Horizonte (Minas Gerais), identificaram elevada proporção de óbitos por asfixia intraparto em fetos com $2.500 \mathrm{~g}$ e mais. Esses levantamentos sugerem que há deficiências no acompanhamento do trabalho de parto na rede SUS.

Este é um estudo exploratório realizado com dados secundários que apresentam algumas limitações. O SINASC apresenta algumas variáveis como número de consultas de pré-natal e idade gestacional, agrupadas em faixas. Ademais, como já mencionado, as informações disponíveis no SIM e SINASC não permitem uma avaliação clara dos riscos presentes nas gestações. Também não foram utilizados indicadores mais sensíveis, como a presença de pediatra na sala de parto e informações sobre o acompanhamento das gestantes durante o trabalho de parto, que permitiriam uma melhor avaliação dos cuidados dispensados às gestantes e aos recém-nascidos. Não estavam disponíveis informações sobre a morbidade de mães e recém-nascidos, e não se avaliaram a presença e o uso de protocolos para esses cuidados. Porém, esses resultados iniciais poderão auxiliar na melhor compreensão da assistência obstétrica e neonatal na Região Metropolitana de São Paulo. 


\section{Resumo}

O objetivo foi analisar o perfil dos recém-nascidos, mães e mortalidade neonatal precoce, segundo complexidade do hospital e vínculo com o Sistema Único de Saúde (SUS), na Região Metropolitana de São Paulo, Brasil. Estudo baseado em dados de nascidos vivos, óbitos e cadastro de hospitais. Para obter a tipologia de complexidade e o perfil da clientela, empregaramse análise fatorial e de clusters. O SUS atende mais recém-nascidos de risco e mães com baixa escolaridade, pré-natal insuficiente e adolescentes. A probabilidade de morte neonatal precoce foi 5,6\%o nascidos vivos (65\% maior no SUS), sem diferenças por nível de complexidade do hospital, exceto nos de altíssima (SUS) e média (não-SUS) complexidade. O diferencial de mortalidade neonatal precoce entre as duas redes é menor no grupo de recém-nascidos $<1.500 \mathrm{~g}(22 \%)$, entretanto, a taxa é $131 \%$ mais elevada no SUS para os recém-nascidos $\geq 2.500 \mathrm{~g}$. Há uma concentração de nascimentos de alto risco na rede SUS, contudo a diferença de mortalidade neonatal precoce entre a rede SUS e não-SUS é menor nesse grupo de recém-nascidos. Novos estudos são necessários para compreender melhor a elevada mortalidade de recém-nascidos $\geq 2.500 \mathrm{~g}$ no SUS

Mortalidade Neonatal Precoce; Sistema Único de Saúde; Serviços de Saúde

\section{Referências}

1. Darmstadt GL, Bhutta ZA, Cousens S, Adam T, Walker N, Bernis L. Evidence-based, cost-effective interventions: how many newborn babies can we save? Neonatal Survival 2. Lancet 2005; 365:977-88.

2. Hallsworth M, Farands A, Oortwijn WJ, Hatziandreu E. The provision of neonatal services: data for international comparisons. Santa Monica: RAND Corporation; 2008.

3. Almeida SDM, Barros MBA. Atenção à saúde e mortalidade neonatal: estudo caso-controle realizado em Campinas, SP. Rev Bras Epidemiol 2004; 7:22-35.

4. Ortiz LP, Oushiro DA. Mortalidade Neonatal no Estado de São Paulo. São Paulo Perspect 2008; 22: 19-29.

5. Almeida MF, Novaes HMD, Alencar GP, Rodrigues LC. Mortalidade neonatal no Município de São Paulo: influência do peso ao nascer e fatores sóciodemográficos e assistenciais. Rev Bras Epidemiol 2002; 5:93-107.

\section{Colaboradores}

Z. P. Silva e M. F. Almeida participaram da concepção e elaboração do estudo, da análise e interpretação dos dados e da elaboração do artigo. L. P. Ortiz, G. P. Alencar, A. P. Alencar, D. Schoeps, E. G. Minuci e H. M. D. Novaes colaboraram na elaboração do estudo e na revisão crítica do artigo.

\section{Agradecimentos}

À Fundação de Amparo à Pesquisa do Estado de São Paulo (FAPESP) e ao Conselho Nacional de Desenvolvimento Científico e Tecnológico (CNPq; processo PP-SUS no. 2006/61304-3) pelo financiamento parcial desse estudo.
6. Martins EF, Velásquez-Meléndez G. Determinantes da mortalidade neonatal a partir de uma coorte de nascidos vivos, Montes Claros, Minas Gerais, 19971999. Rev Bras Saúde Matern Infant 2004; 4:405-12.

7. Weirich CF, Andrade ALSS, Turchi MD, Silva SA, Morais-Neto OL, Minamisava R, et al. Neonatal mortality in intensive care units of Central Brazil. Rev Saúde Pública 2005; 39:775-81.

8. Duarte JLMB, Mendonça GAS. Fatores associados à morte neonatal em recém-nascidos de muito baixo peso em quatro maternidades no Município do Rio de Janeiro, Brasil. Cad Saúde Pública 2005; 21:181-91.

9. Schoeps D, Almeida MF, Alencar GP, França Jr. I, Novaes HMD, Siqueira AAF, et al. Fatores de risco para mortalidade neonatal precoce. Rev Saúde Pública 2007; 41:1013-22.

10. Menezes AMB, Barros FC, Victora CG, Tomasi E, Halpern R, Oliveira ALB. Risk factors for perinatal mortality in an urban area of Southern Brazil, 1993. Rev Saúde Pública 1998; 32:209-16. 
11. Leal MC, Gama SGN, Campos MR, Cavalini LT, Garbayo LS, Brasil CLP, et al. Fatores associados à morbi-mortalidade perinatal em uma amostra de maternidades públicas e privadas do Município do Rio de Janeiro, 1999-2001. Cad Saúde Pública 2004; 20 Suppl 1:S20-33.

12. Lansky S, França E, Cesar CC, Monteiro Neto LC, Leal MC. Mortes perinatais e avaliação da assistência ao parto em maternidades do Sistema Único de Saúde em Belo Horizonte, Minas Gerais, Brasil, 1999. Cad Saúde Pública 2006; 22:117-30.

13. Schramm JMA, Szwarcwald CL, Esteves MAP. Assistência obstétrica e risco de internação na rede de hospitais do Estado do Rio de Janeiro. Rev Saúde Pública 2002; 36:590-7.

14. Novaes HMD. Mortalidade neonatal e avaliação da qualidade de atenção ao parto e ao recém-nascido no Município de São Paulo [Tese de Livre-Docência]. São Paulo: Faculdade de Medicina, Universidade de São Paulo; 1999.

15. Barros AJD, Matijasevich A, Santos IA, Albernaz EP, Victora CG. Neonatal mortality: description and effect of hospital of birth after risk adjustment. Rev Saúde Pública 2008; 42:1-9.

16. Cifuentes J, Bronstein J, Phibbs CS, Phibbs RH, Schmitt SK, Carlo WA. Mortality in low birth weight infants according to level of neonatal care at hospital of birth. Pediatrics 2002; 109:745-51.

17. Heller G, Richardson DK, Schnell R, Misselwitz B, Künzel W, Schmidt S. Are we regionalized enough? Early-neonatal deaths in low-risk births by the size of delivery units in Hesse, Germany 1990-1999. Int J Epidemiol 2002; 31:1061-8.

18. Ribeiro MCSA, Barata RB, Almeida MF, Silva ZP. Perfil sócio-demográfico e padrão de utilização de serviços de saúde para usuários e não-usuários do SUS - PNAD 2003. Ciênc Saúde Coletiva 2006; 11:1011-22.

19. Waldvogel BC, Ferreira CEC, Camargo ABM, Jordani MS, Ortiz Flores LP. Base unificada de nascimentos e óbitos no Estado de São Paulo: instrumento para aprimorar os indicadores de saúde. São Paulo Perspect 2008; 22:5-18.
20. Waldvogel BC. Pesquisa pioneira recupera casos de AIDS no Estado de São Paulo: integração das bases do SINAN-AIDS e da Fundação Seade. Rev Bras Estud Popul 2006; 23:187-90.

21. Mathews TJ, Menacker F, MacDorman MF; Centers for Disease Control and Prevention, National Center for Health Statistics. Infant mortality statistics from the 2002 period: linked birth/infant death data set. Natl Vital Stat Rep 2004; 53:1-29.

22. Aquino TA, Guimarães MJB, Sarinho SW, Ferreira LOC. Fatores de risco para a mortalidade perinatal no Recife, Pernambuco, Brasil, 2003. Cad Saúde Pública 2007; 23:2853-61.

23. Johnson RA, Wichern DW. Applied multivariate statistical analysis. 5th Ed. New Jersey: Prentice Hall; 2001.

24. Ministério da Saúde. Portaria no 1.067 de 4 de julho de 2005. Institui a Política Nacional de Atenção Obstétrica e Neonatal. Diário Oficial da União 2005; 6 jul.

25. Novaes HMD. Pesquisa em, sobre e para os serviços de saúde: panorama internacional e questões para a pesquisa em saúde no Brasil. Cad Saúde Pública 2004; 20 Suppl 2:S147-73.

26. Neumann NA, Tanaka OY, Victora CG, Cesar JA. Qualidade e eqüidade de atenção ao pré-natal e ao parto em Criciúma, Santa Catarina, Sul do Brasil. Rev Bras Epidemiol 2003; 6:307-18.

27. Minuci EG, Almeida MF. Diferenciais intra-urbanos de peso ao nascer no Município de São Paulo. Rev Saúde Pública 2009; 43:256-66.

28. Tanaka ACA. Maternidade: dilema entre nascimento e morte. São Paulo: Editora Hucitec/Rio de Janeiro: ABRASCO; 1995.

29. Morais Neto OL, Barros MBA. Fatores de risco para mortalidade neonatal e pós-neonatal na Região Centro-Oeste do Brasil: linkage entre bancos de dados de nascidos vivos e óbitos infantis. Cad Saúde Pública 2000; 16:477-85.

Recebido em 11/Fev/2009

Versão final reapresentada em 27/Ago/2009

Aprovado em 22/Set/2009 